\title{
Uma Discussão Teórica sobre Aprendizagem, Inovação e Cultura nos Arranjos e Sistemas Produtivos Territoriais
}

\section{Theoretical Schemes about Learning, Innovation and Culture in the Territorial Arrangements and Productive Systems}

Ana Sílvia Rocha Ipiranga ${ }^{1}$

\begin{abstract}
Resumo
Na atual economia da aprendizagem (learning economy), as redes interempresariais são espaços não só de compartilhar o conhecimento tácito (associado ao learning by doing), os quais acentuam a aprendizagem como um processo localizado que ocorre por meio da interação e da imbricação social, mas também de consolidar esses territórios produtivos como os mais adequados para promover a geração, a aquisição e a difusão de inovações. Por outro lado, os conhecimentos tácitos são disseminados no território dos arranjos e sistemas produtivos, através dos canais de cooperação que estão baseados no compartilhamento de valores culturais. Ao considerar as modalidades de aprendizagem e a análise do conhecimento que vem gerado e disseminado nas redes de inovação, este ensaio tem como objetivo articular conceitos e esquemas teóricos que poderão servir de guia para o reconhecimento do complexo fenômeno da aprendizagem e da inovação em uma organização estruturada, mas informal, como um arranjo ou sistema produtivo territorial. Ao final, conclui-se que a discussão sobre a capacidade de inovação e de produção de inteligência coletiva nos territórios produtivos tem possibilitado uma reorientação na formulação de políticas públicas em prol do desenvolvimento local.
\end{abstract}

Palavras-chave: Arranjos e Sistemas Produtivos Locais, Aprendizagem, Inovação e Cultura.

\begin{abstract}
In the current learning economy, the networks between companies are spaces of sharing tacit knowledge, associated to the 'learning by doing', accentuating learning as a localized process that takes place through interaction and social imbrication, consolidating these productive territories as the most adequate to promote the generation, acquisition and diffusion of innovation. On the other hand, the tacit knowledge is disseminated on the territory of arrangements and productive systems through the channels of cooperation that are based on the sharing of cultural values. This essay, when considering the learning modalities and the analysis of the knowledge that comes generated and disseminated in the networks of innovation, aims at articulating concepts and theoretical schemes that will be able to act as guidelines to acknowledging the complex phenomenon of learning and of innovation in a structured organization, but informal as a territorial arrangement or productive system. Ultimately, it is considered that this discussion on the capacity of innovation and production of collective intelligence in the productive territories has made possible the re-orientation in the formulation of public policies that favor local development.
\end{abstract}

Keywords: Local Arrangements and Productive Systems, Learning, Innovation, Culture.

${ }^{1}$ Mestrado Acadêmico em Administração. Coordenadora do Laboratório Tecnologias Digitais e Pesquisa Qualitativa Universidade Estadual do Ceará - Endereço: Av. Paranjana, 1700, Campus do Itaperi - Fortaleza-CE - Brasil. CEP.: 60740-000. E-mail: anasilviaipi@uol.com.br.

Artigo submetido em outubro de 2006 e aceito em janeiro de 2007. 


\section{Introdução}

$\mathrm{Na}$ atual economia da aprendizagem (learning economy), as aglomerações produtivas territoriais são espaços não só de compartilhar o conhecimento tácito (associado ao learning by doing) os quais acentuam a aprendizagem como um processo localizado que ocorre por meio da interação e da imbricação social, fortalecendo as redes e práticas de inovação.

Dentre as abordagens que se propõem analisar a organização das pequenas e médias empresas com foco no território, encontram-se aquelas - foco deste estudo - denominadas arranjos produtivos locais (APLs), 1 que representam um estágio de transição para formas mais sistêmicas e com maior nível de interdependência entre os atores/agentes, os assim chamados sistemas produtivos locais (SPLs)2. De acordo com Cassiolato e Szapiro (2002), a abordagem do arranjo e sistema produtivo local tem a proposta de elaborar um modelo que englobe categorias tradicionais nas análises de aglomerações como a cooperação, mas que inclua, também, os processos de aprendizado e inovação, considerados crescentemente como fundamentais para a sustentação da competitividade dos agentes participantes dessas aglomerações produtivas territoriais.

Segundo a literatura, em cada arranjo e em cada sistema produtivo territorial, a performance competitiva, individual, ocorre em modalidades diversas e específicas, mas alguns elementos comuns podem ser identificados como, por exemplo, a existência de um ambiente (social, institucional, produtivo e tecnológico) que necessite da interação contínua e repetida entre as empresas e os atores/agentes; a existência de uma pluralidade de redes relacionais (formais e informais), entre os atores/agentes do sistema, consentindo um eficiente compartilhamento de informações, transparência de comportamentos, etc.; a existência de uma relevância atribuída ao reconhecimento social do ato de empreender; a existência de uma definição endógena dos limites do sistema, principalmente no sentido da participação e da pertença dos atores/agentes envolvidos.

Por outro lado, a performance competitiva dos arranjos e sistemas produtivos territoriais é geralmente invocada de forma implícita e indireta na literatura empírica sobre o 'capital social' (BOURDIEU, 1985; COLEMAN, 1988; PUTNAM, 1996) enquanto "expectativa generalizada de cooperação" (BERETTA; CURINI, 2003) e da 'governança' que se refere ao poder compartilhado através da gestão da ação coletiva, desencadeando processos de aprendizagem e influenciando o desenvolvimento da "inovação social" (MESSNER; MEYERSTAMER, 2000). Nesse sentido, a 'cultura' dos arranjos e sistemas produtivos territoriais envolve os valores compartilhados, a cooperação e o capital social.

Para Lundvall (2002) a presença e a forma do 'capital social' num contexto mais amplo afetará o processo de aprendizagem, pois os atores/agentes, ao privilegiarem a interação e a atuação conjunta, consolidam esses territórios produtivos como os mais adequados para promover a geração, a aquisição e a difusão de conhecimento e inovações. O locus do aprendizado deixa de ser exclusivamente a firma individual e passa a incluir, também, a interface com outros atores/agentes presentes no território. $\mathrm{O}$ foco de análise centra-se nas relações entre as firmas e entre essas e as instituições (organizações e agências públicas e privadas, ONGs, universidades e centros de pesquisa, associações de classe e comunitárias) que interagem com elas num determinado espaço territorial. A unidade de análise mais apropriada para a compreensão desse ambiente seria o arranjo e o seu território.

De modo geral, os estudiosos da economia da inovação (FREEMAN, 1982) acreditam que para haver inovação é preciso que determinados fatores do meio ambiente sejam propícios àquela ocorrência. Segundo Rothwell (1995) a percepção do modelo de inovação dominante e, por extensão, das práticas inovadoras tem passado por algumas mudanças, que podem ser identificadas por diferentes gerações do processo inovador. Para o autor, atualmente, o processo inovador passa a ser um sistema integrado em uma ampla rede de cooperação. Neste âmbito, Lundvall (2002) ressalta a importância de olhar a dimensão social e política do conceito de inovação. A capacidade de inovação deriva da confluência de fatores sociais, institucionais e culturais específicos aos ambientes em que se inserem os atores/agentes; além da performance competitiva dos setores nos quais as empresas estão inseridas (CALLON, 1992).

As redes de cooperação interempresariais, ao facilitar a difusão da informação e o compartilhar de conhecimento, podem ser significativas no contexto tecnológico (POWELL et al., 1996), pois a inovação é 
freqüentemente uma atividade intensiva em informação e conhecimentos externos à pequena e média empresa (VEDOVELLO et al., 2004). No ambiente dessas arquiteturas de relacionamento, a ' capacidade de inovação' se desenvolve na fluida mobilidade entre os arranjos, as empresas e as pessoas - e no interior destes. Como um canal privilegiado de comunicação, circulação, processamento da aprendizagem coletiva através da troca de experiências e de conhecimentos contextuais (não codificados e nem transferíveis), tal mobilidade se exprime, representando, por fim, a base 'tecnológica' dos arranjos e sistemas produtivos territoriais.

Este ensaio tem como objetivo articular conceitos e esquemas teóricos que poderão servir de indicações para o reconhecimento do complexo fenômeno da aprendizagem e da inovação em uma organização estruturada, mas informal, como um arranjo e sistema produtivo territorial. No primeiro item coloca-se em discussão a base 'tecnológica' dos arranjos e sistemas produtivos, definindo-se, em um segundo momento, as modalidades de aprendizagem e de inovação ativadas nestes ambientes. Considerando-se a análise do conhecimento que vem gerado e disseminado nas redes de inovação, no segundo item, se demonstra como essas interações influenciam tanto a criação de conhecimentos como também a sua natureza, gerando, conforme os casos, conhecimentos culturais, tácitos e codificados (ou explícitos). No terceiro item se descrevem possíveis cenários de interação, articulados através das práticas e das conexões alternativas da aprendizagem coletiva, verificando-se as implicações para a geração e difusão da inovação. No último item se apresentam algumas reflexões sobre o trabalho.

\section{Contexto Tecnológico dos Arranjos e Sistemas Produtivos Territoriais}

Partindo-se de uma visão sistêmica, os arranjos e sistemas produtivos territoriais devem ser analisados, considerando-se três vertentes que, juntas, contribuem para o incremento da competitividade e para os avanços da sustentabilidade: a) o desenvolvimento da capacidade produtiva e inovadora; b) o fortalecimento do capital social e da governança; c) o aprendizado e a formação de competências territoriais e dos seus atores/agentes.

Especificamente, quanto à vertente 'formação de competências', salienta-se que a 'competência' que se busca corresponde à 'inteligência prática', que implica saber como mobilizar, integrar e transferir os conhecimentos, recursos e habilidades em um contexto determinado (ZARIFIAN, 2001). No âmbito dessa discussão sobressaise o desenvolvimento das 'competências tecnológicas'.

As competências tecnológicas devem ser entendidas como o conjunto de recursos necessários para gerar e gerir mudanças tecnológicas, incluindo habilidades, conhecimentos, experiências e sistemas organizacionais. Competências tecnológicas referem-se especificamente à habilidade das empresas de realizarem internamente melhorias no processo e na organização da produção, nos produtos, nos equipamentos e nos projetos de engenharia (BELL; PAVITT, 1995; VEDOVELLO et al., 2004).

No âmbito dos arranjos e sistemas produtivos territoriais, dentre tais recursos destacam-se o conhecimento tácito, codificado (ou explícito) e cultural - e ainda, as estruturas, ligações e relações produtivas, comunitárias e institucionais que conformam o território vivido pelos atores/agentes. Especificamos, a seguir, cada conjunto desses recursos e dimensões.

Segundo Throsby (2001, p.4), o termo 'cultura' pode ser entendido em dois significados diferentes, nem sempre alternativos. O primeiro, de base funcionalista, refere-se a um conjunto específico de "atividades e de produtos de tais atividades, relacionados aos aspectos intelectuais, morais e artísticos da vida humana". Nesse sentido, o termo cultura, está associado mais às atividades ligadas "à iluminação e à educação da mente e menos à aquisição de capacidades técnicas ou práticas". O segundo, de matriz antropológica e sociológica, descreve a cultura como "uma teia de significados, atitudes, usos, costumes, valores e práticas que são comuns ou compartilhadas por um grupo definido em termos políticos, geográficos, religiosos, étnicos ou de outras características".

Essas duas definições são aqui consideradas para sublinhar a importância do papel da 'cultura', tanto no sentido etnológico quanto sociológico do termo, ao estimular o desenvolvimento local, econômico e tecnológico nos territórios dos arranjos e sistemas produtivos. 
Para Throsby (2001) é possível classificar as influências da cultura na performance competitiva de uma entidade - no nosso caso, um arranjo ou sistema produtivo territorial - em três grandes categorias:

a) A cultura pode influenciar a eficácia do arranjo territorial seja através da identificação de alguns objetivos sociais e econômicos que se pretende alcançar, seja através da construção de práticas sociais e normas compartilhadas que permitam o alcance de um equilíbrio mais homogêneo no âmbito das relações interpessoais e coletivas;

b) A cultura poderá, também, influenciar a eficiência de um arranjo através da promoção de valores compartilhados, condicionando as modalidades do processo produtivo em nível da empresa individual e, sobretudo, do inteiro arranjo, como, por exemplo, diminuindo os custos de transação;

c) Enfim, a cultura, ainda segundo Throsby (2001), pode influenciar a eqüidade de um arranjo territorial, seja através da legitimação e da transmissão de princípios morais, direcionados a valorizar a interação em relação aos outros participantes do arranjo, seja através da construção de mecanismos sociais e institucionais através dos quais possa exprimir tais interações e princípios, seja, por exemplo, na promoção de uma 'boa' governança (IPIRANGA et al., 2004).

Além do conhecimento cultural, que, por sua vez, é formado por suposições e crenças que são utilizadas para descrever e explicar a realidade e para dar valor e importância a cada nova informação (CHOO, 1998), no âmbito dos arranjos e sistemas produtivos territoriais, destaca-se, ainda, o conhecimento tácito e codificado (ou explícito). Este último é a parte mais estruturada do conhecimento, facilmente registrada e comunicada em oposição ao conhecimento tácito (POLANYI, 1966), que é a parte menos estruturada, composta de sensações, rotinas, experiências, imagens que não deram origem a enunciados claros na mente e são, assim, dificilmente comunicados.

Em relação às estruturas, ligações e relações que conformam os territórios produtivos, sobressaem-se, no campo das políticas públicas, os conceitos de "Política de Inovação e Sistema Nacional de Inovação ", que se tornaram a base de explicação para as iniciativas dos governos que buscam apoiar a criação de pequenas empresas de alta tecnologia, a transferência de conhecimentos entre universidades, centros de pesquisa e empresas, a criação de programas e de redes estáveis de trocas de informações e conhecimentos (LUNDVALL, 1992).

Lundvall (2002) propõe trabalhar o conceito de Sistema de Inovação em duas dimensões: uma que se refere à estrutura do sistema - o que se produz e quais competências são desenvolvidas; e a outra, à dimensão institucional - como a produção, inovação e aprendizado acontecem. Segundo o autor, a análise histórica e cultural é fundamental na percepção de como essas duas dimensões co-evoluem. Lundvall salienta, ainda, que o processo de aprendizagem é socialmente vinculado e, que a iniciativa de organizações e instituições são cruciais para o surgimento das interações e do intercâmbio.

Essa perspectiva se coaduna com a colocação de Rothwell (1995) quando acentua que a inovação é uma ação conjunta e cooperada de diversos atores, públicos e privados, internos e externos ao arranjo e sistema produtivo. As redes de cooperação entre as empresas, ao facilitar a difusão da informação e o compartilhamento de conhecimento, podem ser significativas no contexto tecnológico (POWELL et al., 1996), pois a inovação é freqüentemente uma atividade intensiva em informação e em conhecimentos externos à pequena e média empresa. Nesse sentido, o desenvolvimento e a acumulação de competências tecnológicas são fundamentais para que um arranjo ou sistema produtivo torne-se capaz de realizar 'atividades inovadoras' (VEDOVELLO et al., 2004).

Ao tratar das competências tecnológicas, faz-se necessário que sejam distinguidos os conceitos de 'capacidade de produção' e 'capacidade da inovação'. Para isto, deve-se considerar a diferença entre as "competências de rotina - competências para usar - e as competências inovadoras - competências para mudar" (BELL; PAVITT, 1995). A 'capacidade de produção' relaciona-se com as 'competências de rotina', que são os recursos para produzir bens e serviços, utilizando-se, para isso, de uma combinação de fatores: habilidades, equipamentos, especificações de produtos, sistemas e métodos organizacionais. Já a 'capacidade de inovação' incorpora 
recursos adicionais e distintos para gerar e gerir a mudança tecnológica (BELL; PAVITT, 1995; VEDOVELLO et al., 2004).

Para Vasconcelos (2001), as 'inovações' - enquanto transformações de uma descoberta em uma nova prática podem ser vistas como re-configurações de sistemas de atividade econômica, como mudanças de papéis que os atores econômicos desempenham ao interagir em sistemas de criação de valor. O autor diferencia as 'microinovações' das 'macro-inovações'. As primeiras são 'inovações incrementais' e se relacionam à eficiência das operações atuais, sendo importantes para atingir ganhos operacionais e prover melhorias contínuas nos sistemas de produção. As 'macro-inovações' buscam alternativas para o futuro, sendo fundamentais por permitirem saltos qualitativos nos sistemas produtivos, nas posições competitivas das empresas e nas configurações da arquitetura de relacionamentos entre os atores econômicos. Para o autor, estas últimas são 'inovações radicais' que criam uma nova configuração de atores econômicos. Estão ligadas à criação de valores qualitativamente novos e implicam a constituição de novas redes de atores que colaboram para criar valor ou constelações de valor.

A 'capacidade de inovação', no contexto dessas arquiteturas de relacionamentos, se desenvolve na fluida mobilidade entre os arranjos, as empresas e as pessoas e no interior desses. Tal mobilidade se exprime como um canal privilegiado de comunicação, circulação, processamento da aprendizagem coletiva através da troca de experiências e conhecimentos contextuais (não codificados e nem transferíveis), representando, por fim, a base 'tecnológica' dos arranjos e sistemas produtivos territoriais.

\section{Modalidades de Aprendizagem e Inovação nos Arranjos e nos Sistemas Produtivos Territoriais}

Em relação à análise das práticas e implicações do processo de aprendizagem e, considerando as contribuições seminais sobre as atividades de 'aprendendo fazendo' (learning by doing) (ARROW, 1962), 'aprendendo usando' (learning by using) (ROSENBERG, 1982 ) e 'aprendendo a aprender' (learning to learn) (STIGLITZ, 1987), uma boa quantidade de pesquisas foi desenvolvida, guiada por objetivos que se deslocavam entre diferentes espaços disciplinares - da sociologia à psicologia, da organização à administração.

Considerando os objetivos do presente ensaio propõe-se o esquema de Lipparini e Lorenzoni (1996) que poderá servir de guia para o reconhecimento do complexo fenômeno da aprendizagem em uma organização estruturada, mas informal, como um arranjo e sistema produtivo territorial.

Conforme Lipparini e Lorenzoni (1996), tendo por base as relações de conteúdo e de nível de relação entre empresas e, até certo ponto, da abertura para o externo do arranjo (como sugerido por Bramanti e Maggioni, 1997; Maggioni e Bramanti, 2000), é possível definir as seguintes modalidades de aprendizagem e inovação:

1. A aprendizagem da localização (Learning by localising) - associada à abordagem "clássica dos distritos industriais italianos" como a de Marshall (1996), Beccattini (1987, 1989) e Brusco (1986) nas quais pertencer a um arranjo, entendido como 'ambiente', representa a condição 'natural' para aprender a fazer ou para melhorar os conhecimentos já desenvolvidos pelas empresas (LIPPARINI; LORENZONI, 1996 p.820).

O termo chave na dinâmica da aprendizagem é aquele 'marshalliano' de "atmosfera industrial," que evidencia o caráter de não intencionalidade das relações e de estreito caráter local da atmosfera, se configurando como um bem não rival, mas certamente excludente. Nesse sentido, a aprendizagem da localização é uma vantagem competitiva no confronto das empresas não localizadas, agindo como uma barreira à entrada para os concorrentes que não operam em iguais condições de informação e coordenação.

A inovação conseqüente a essa modalidade de aprendizagem tem características principalmente incrementais, limitadas às aplicações no setor dominante do arranjo através das interações entre produtores e fornecedores.

2. A aprendizagem da especialização (Learning by specialising) associada à abordagem da especialização flexivel (PIORE; SABLE, 1984; AMIN, 1991) e do "Industrial Divide" - na qual o 
acento é colocado sobre as características de especialização e de flexibilidade entendida como rápida capacidade de resposta a mudanças tecnológicas e a choques exógenos da demanda.

Nesse contexto, a inovação emerge através da interação entre as empresas em diferentes fases da cadeia produtiva, contemporaneamente de acordo com as complementaridades técnicas e produtivas, construídas nas relações com os fornecedores. Por conta da natureza tecnológica dessas relações, esse modelo foi adotado para descrever dinâmicas de organização da produção como, por exemplo, as redes de empresas locais que se configuraram a partir das estratégias de descentralização produtiva e / ou de re-organização de grandes empresas.

3. A aprendizagem coletiva (Collective learning) - associada à abordagem GREMI ou do "milieu innovateur" (AYDALOT; KEEBLE, 1998; CAMAGNI, 1991; MAILLATT et al., 1993; RATTI et al., 1997) - na qual o contexto do arranjo e sistema territorial age como redutor sistêmico de incertezas nos processos inovadores, criadores de redes de interações e de convenções, normas de comportamentos e códigos compartilhados de inclusão / exclusão.

Neste contexto a aprendizagem coletiva vem definida como aprimoramento do conhecimento de acordo com uma trajetória tecnológica, incorporada em um contexto local que acontece através de específicos canais, como, por exemplo, relações estáveis entre clientes e fornecedores locais (CAMAGNI; CAPELLO, 2002), e, ainda, a presença de um mercado local de trabalho caracterizado de baixo turnover externo e elevada mobilidade interna com elevados mecanismos de spinoff e start-up imitativos de empresas locais.

O resultado final dessa aprendizagem pode ser medido no âmbito do sistema local, através da performance em termos de crescimento das competências de trabalho e do nível da ocupação, na presença de inovações de produtos, de processos e de organização e, por fim, na diversificação e modernização setorial da estrutura produtiva.

4. A aprendizagem da interação (Learning by interating) - associada à abordagem 'empresa-centro' para os arranjos e sistemas locais (LORENZONI, 1990 e 1992; LIPPARINI; LOMI, 1998; FERRUCCI; VARALDO, 1993; LIPPARINI; LORENZONI, 1996) - na qual, ao lado dos fatores ambientais, se evidencia o papel de fatores empreendedores (em particular o papel guia assumido por alguns atores chaves) na promoção e sustenho do processo inovador.

Nesse contexto, a aprendizagem da interação implica a deliberação de ações para a organização e o funcionamento da arquitetura relacional do sistema, pressupondo a sua abertura para o ambiente externo. Interagindo com os fornecedores, com os clientes, e também com os concorrentes, é possível melhorar aquilo que já se estava fazendo (segundo uma lógica incremental) e aprender a fazer coisas novas (segundo uma lógica radical do processo inovador). Mas, conforme os autores, a capacidade relacional não exaure o inteiro processo de aprendizagem da interação. Esse processo pressupõe, também, a capacidade de absorção das informações e dos conhecimentos adquiridos através das interações; da capacidade de combinação das informações e dos conhecimentos internos e externos e, por fim, da capacidade de coordenação do conjunto relacional (governança) e dos mecanismos de transferência dos conhecimentos.

\section{Conhecimento Cultural, Tácito e Codificado nos Arranjos e Sistemas Produtivos Territoriais}

As modalidades de aprendizagem acima ilustradas solicitam a análise do conhecimento que vem gerado e disseminado nas redes de inovação (de práticas sociais) constituídas entre os atores/agentes dos arranjos e sistemas produtivos territoriais. Por outro lado, essas análises sobre os processos de aprendizagem no contexto do arranjo demonstram como essas interações entre os agentes presentes nos territórios produtivos influenciam tanto a criação de conhecimentos como também a sua natureza, gerando, conforme os casos, conhecimentos culturais, tácitos e codificados (ou explícitos). 
Evidenciando a relevância que a inovação assume na era do conhecimento, alguns autores destacam que é necessária uma nova teoria de criação do conhecimento para explicá-la. Conforme anteriormente colocado, os primeiros estudos sobre a dupla natureza dos conhecimentos, tácitos e codificados, são referidos nos trabalhos de Polanyi (1958) onde através da expressão "nós sabemos mais do que aquilo que imaginamos" (POLANYI, 1966) o autor indicou que o conhecimento tácito representa tudo aquilo que não pode ser expresso diretamente através de palavras ou de números que, por sua vez, definem o conhecimento codificado.

Aprofundando a discussão, o conhecimento tácito é constituído de intuições, sensações, idéias, capacidades difíceis de transmitir por estarem profundamente radicadas nas práticas e experiências individuais e fortemente conectadas a valores e ideais compartilhados por grupos específicos de pessoas. Segundo essa perspectiva, o conhecimento tácito pode ser subdividido em duas componentes: uma técnica e uma cognitiva. A primeira compreende as competências e as habilidades possuídas por quem desenvolve atividades com elevado conteúdo de conhecimentos; a segunda relaciona-se aos aspectos ideais e valorais não facilmente identificáveis, mas que determinam os modos de relacionar-se dos indivíduos, modos esses identificados com a cultura local.

A dimensão codificada do conhecimento é expressa em modo formal e sistemático e, como tal, só é transmitida sob a condição do compartilhamento dos códigos através dos quais foi explicitada.

Conforme a classificação de Johnson e Lundvall (2001), o conhecimento de fatos ou informações - 'o saber o que' - e o conhecimento dos princípios das regras e das idéias - 'o saber o porquê' - fazem parte dos conhecimentos reproduzíveis e, portanto, codificados. A habilidade no fazer alguma coisa por experiência direta - 'o saber como' - e o conhecimento de quem sabe fazer alguma coisa, a capacidade de comunicação e de construir relação de confiança - 'o saber quem' - constituem os conhecimentos adquiridos através da prática e da experiência ou de contatos diretos em contextos particulares - empresas, associações, arranjos e sistemas locais - e por isso considerado tácitos.

O 'individuo', a 'empresa' e o 'contexto' constituem a chave de leitura através da qual é possível aprofundar os diversos temas conectados aos conhecimentos cultural, tácito e codificado.

O 'individuo' constitui o primeiro e fundamental nível de consideração para se compreender a aquisição e disseminação de conhecimentos. A criação de conhecimentos, em particular aqueles tácitos, parte do indivíduo para depois se disseminar na organização e no contexto.

Kim (1993) caracteriza a aprendizagem individual como aquela que ocorre a partir da experiência, da observação e da capacidade que o indivíduo tem de refletir e avaliar a situação, gerando estruturas cognitivas, modelos interpretativos, práticas e rotinas pessoais de trabalho. A tensão que se estabelece nos estudos sobre aprendizagem organizacional relaciona-se com a demarcação nítida entre os dois primeiros níveis de ocorrência desse fenômeno - individual e organizacional. Apesar dos esforços em tratar essa questão, tal demarcação ainda não foi alcançada; entretanto muitos autores lançam possíveis articulações acerca desse tópico.

Nesse âmbito, a 'empresa' apresenta o segundo nível de criação e geração de conhecimentos. Uma relevante contribuição para a compreensão da importância da empresa no processo de knowledge creation se origina das pesquisas de Nonaka e Takeuchi (1995) sobre o papel das organizações em criar o conhecimento, disseminar e traduzi-lo em produtos e serviços. Os autores destacam, ainda, que a criação do conhecimento seja a mais importante fonte de inovação em qualquer organização, e que parece existir forte relação entre inovação e conhecimento tácito, sendo que, em geral, esse tipo de conhecimento representa a sua fonte de vantagem competitiva.

Os processos organizacionais fazem referências às atividades que, através do recurso da 'resolução de problemas' (learn by doing), consentem galgar níveis de conhecimentos superiores que conduzem à inovação e à criação de vantagens competitivas. Nessa visão é fundamental o incremento de conhecimentos que derivam da contínua relação do individuo com a organização e do contínuo confronto de ambos com a realidade externa. 
Resumindo-se os resultados dessa abordagem, é possível afirmar que a observação daquilo que ocorre fora da empresa estimula a inovação por meio de um contínuo processo de interiorização e elaboração, incrementando as vantagens competitivas da empresa individual e do sistema integrado no qual esta está inserida.

Por outro lado, a visão interpretativa tem considerado o conhecimento como amplamente arraigado nas práticas e processos organizacionais, tendo como base o princípio do conhecimento socialmente construído por meio da interação entre os indivíduos, grupos e organizações. Considerando essa linha de pensamento e conforme Nonaka, Toyama e Konno (2002), um fator estratégico para a organização é a potencialidade em criar novos conhecimentos.

O processo de criação e interação dos conhecimentos entre empresas e contextos parte da "socialização" que consente compartilhar aspectos tácitos do conhecimento individual por meio da observação, imitação, práticas e modelos mentais, favorecendo a construção de um "campo de interação", um espaço de colaboração entre sujeitos. Em seguida, com a "exteriorização" se coloca o problema de formalizar, explicitando o quanto possível, os conhecimentos tácitos já compartilhados. Isso ocorre, segundo Nonaka e Takeuchi (1995), com o uso da linguagem figurada (metáforas, analogias, simbolismos) e através de instrumentos como o diálogo e a reflexão coletiva para articular aquilo que é de difícil expressão. O terceiro ciclo é aquele da 'combinação' de conhecimentos, inclusive externos à organização e da sua aplicação em produtos e serviços inovadores. Isso se dá através da articulação do conhecimento por meio de documentos, reuniões, conversações ou rede de comunicação e através da ligação entre conhecimentos tácitos e explícitos. A quarta e última fase relaciona-se à 'interiorização' dos conhecimentos, consentindo uma nova contextualização dos conhecimentos codificados e a maturação de novos conhecimentos tácitos; processos relacionados ao 'aprender fazendo' e que ocorrem mediante a aprendizagem através da 'prática' e da 'experiência'.

Para que o conhecimento explícito se torne tácito é necessário verbalização e diagramação do conhecimento sob a forma de documentos, manuais ou histórias orais. A "espiral do conhecimento" (NONAKA; TAKEUCHI, 1995) a esse ponto se completa através da produção de novos conhecimentos que passam a dar início a um novo processo, permitindo, enfim, a compreensão do processo de criação de conhecimento a partir da interação contínua entre as dimensões tácitas e codificadas (ou explícitas) do conhecimento e entre o indivíduo e o grupo. Por conseguinte, a sinergia entre os processos de criação e transferência de conhecimento, ressalta a importância do grupo (dimensão social) na criação (individual) do conhecimento. Nesse processo, o papel reservado às organizações é o de propiciar um contexto compartilhado - um espaço físico, virtual ou mental, dentro do qual o conhecimento é gerado, partilhado e utilizado - possibilitando o engajamento e a interação dos indivíduos em um constante diálogo.

O terceiro nível é aquele do 'contexto', que se constitui como um ponto de encontro das diversas abordagens que, segundo perspectivas diferentes, tentaram explicar a relação existente entre território e natureza do conhecimento.

No âmbito dos arranjos e sistemas produtivos, a criação e a disseminação de conhecimentos ocorrem através de um processo contínuo de integração entre os conhecimentos que fazem parte do patrimônio cultural do território do arranjo. Conforme Lundvall e Maskell (2000), a experiência histórica e a cultura se refletem em questões importantes para o processo de aprendizagem e inovações tais como a organização interna das firmas, as relações interfirmas, o papel do setor público, o arcabouço institucional do setor financeiro, etc.

Os conhecimentos típicos dos arranjos e sistemas de produção constituem a parte mais importante daquilo que foi definido como "capacidades territoriais" (regional capabilities) (MASKELL; MALMBERG, 1997) ou "territórios de aprendizagem" (learning regions) (LUNDVALL; MASKELL, 2000). De fato a proximidade espacial, segundo estes autores, se transforma na chave para a produção, a transferência e o compartilhamento dos conhecimentos tácitos gerados em conjunto, salientando a importância do contexto social e cultural para este tipo de aprendizado.

Corroborando tal pensamento, Cowan et al. (1998) indicam que os benefícios advindos dos conhecimentos tácitos estão disponíveis e podem ser colhidos apenas localmente. O conhecimento tácito é inseparável do lugar onde foi gerado e daqueles que o geraram. A forte ligação com o contexto e a escassa comercialização dos 
conhecimentos tácitos determinam que esses não podem ser erodidos nem da competição nem da mobilidade da informação e das pessoas. Dada a dificuldade de serem trocados, assumem ainda maior relevância se são analisados à luz da competição internacional, no momento em que se colocam como um dos componentes fundamentais de vantagem competitiva local em escala global (MAGGIONI; NOSVELLI, 2003).

Por fim, sublinha-se que, apesar dessas características, os conhecimentos tácitos podem ser disseminados no território dos arranjos e sistemas produtivos através dos canais de cooperação, baseados no compartilhamento de valores e cultura, características essas da "atmosfera do distrito" segundo Marshall (1996) e Becattini (1989).

Enfim, o aspecto cultural, junto com o da proximidade espacial, se apresenta como fator crucial para a criação, localmente, dos conhecimentos tácitos. A existência de um substrato cultural comum é associada à existência de uma relação de confiança (capital social) que justifica a transmissão dos conhecimentos tácitos para os outros. Se, por um lado, a cultura entra como pré-requisito para a criação de conhecimentos codificados, por outro, representa um aspecto fundamental para a geração e difusão dos conhecimentos tácitos.

\section{Cenários de Interação: aprendizagem coletiva e a geração e difusão das inovações nos arranjos e sistemas produtivos territoriais}

A questão sobre o que é local / interno e o que é global / externo é central tanto para a definição das modalidades de aprendizagem e de inovação como para determinar a performance competitiva de um arranjo ou sistema produtivo territorial.

Grande parte da literatura insiste em acentuar que, para desenvolver-se um arranjo, se deve balancear dinamicamente o grau de vitalidade interna do seu tecido (sinergia interna) com o de abertura para o mundo (participação em redes globais ou em relações externas).

Por outro lado, evidencia-se que qualquer solução desequilibrada (verso o externo ou verso o interno) leva, em médio ou longo prazo, à crise do sistema local ou à "morte por entropia", devido a um mero crescimento numérico das empresas e a comportamentos empreendedores imitativos. Tal distorção expõe o sistema ao risco de choques idiossincráticos, desencadeando uma destrutiva competição de preços, ou ainda a uma desintegração do sistema, devido à 'deslocalização' de algumas das fases da cadeia produtiva e das atividades manufatureiras, com a conseqüente perda dos efeitos espaciais, sobretudo relacionais, da proximidade (BRAMANTI; MIGLIERINA, 1995).

Considerando essas discussões, alguns pesquisadores (MAGGIONI; RIGGI, 2002) desenvolveram estudos, simulando diversos cenários de interação, através dos dois canais alternativos de aprendizagem coletiva - "as conexões internas e as conexões externas" - para verificar as implicações sobre o processo de criação e de difusão da inovação que se nutre do tecido (social, cultural, institucional, tecnológico e produtivo) no qual estão localizadas as empresas.

A essas duas alternativas de conexões (internas e externas) é possível associar as duas tipologias de conhecimento - o conhecimento tácito e o conhecimento codificado (ou explícito) - dado que transferências de conteúdo, informação e conhecimento, do externo para o interno do sistema, exigem qualquer codificação; enquanto que transferências de conteúdo, informação e conhecimento do interno para o externo, ocorrem através do compartilhamento das rotinas e práticas das atividades produtivas.

Considera-se, ainda, que as duas diferentes tipologias de aprendizagem façam referência aos dois significados atribuídos ao termo cultura segundo Throsby (2001), no início desse ensaio. O conhecimento tácito, veiculado através das conexões internas, se refere à "cultura do arranjo" enquanto um conjunto de valores compartilhados e às expectativas de cooperação. O conhecimento codificado, veiculado através das conexões externas, necessita de certo "nível cultural", isto é, de certo grau de instrução formal, mínimo, para poder ser recebido, adaptado e adotado proficuamente pelos atores/agentes do arranjo ou sistema territorial. 
Para o primeiro caso (conexões internas, modalidade endógena de aprendizagem, conhecimento tácito), os autores formularam a hipótese de que a inovação é gerada (através de um procedimento casual periódico) ao nível interno de uma empresa individual e a hipótese de que, após, se difunde no arranjo, através das redes de inovações originadas das interações existentes entre as empresas. A velocidade de difusão da inovação será proporcional à facilidade, freqüência e qualidade das interações sociais, econômicas, tecnológicas e produtivas entre inovadores e potenciais adotantes.

As interações entre inovadores e potenciais adotantes ocorrem com base em um parâmetro, que depende da capacidade inovadora das empresas individuais, da densidade relacional, do grau de confiança recíproca e da colaboração existente ao interno do arranjo ou sistema territorial. Estando ainda relacionado com o produto que resulta do número de inovadores (ou aqueles que já adotaram a inovação) e do número de potenciais adotantes. Dessa forma determina-se o processo de inovação - gerado por mecanismos endógenos de aprendizagem segundo um percurso de expansão sigmóide cuja 'pendência' é regulada pelo citado parâmetro exógeno.

No segundo caso (conexões externas, modalidade exógena de aprendizagem, conhecimento codificado) foi considerada a hipótese de que a produção no arranjo acontece segundo modalidades tradicionais e imutáveis no tempo e que somente o contato esporádico com o externo permite às empresas aprender e melhorar sua performance. Esse contato acontece somente quando a empresa se aproxima de uma "janela tecnológica" (technological windowing) por um período de tempo suficiente para observar, imitar e assimilar a inovação.

Nesse caso, a taxa de inovação é positivamente influenciada pelo número de empresas presentes no arranjo e potencialmente dispostas a inovar. Um exemplo desse segundo caso muito recorrente no Brasil acontece quando as empresas de um arranjo formam consórcios para organizar eventos como a participação do setor específico da atividade do arranjo ou sistema produtivo em uma feira tecnológica no estrangeiro (ou mesmo nacional, mas externa ao território do arranjo).

Nessa modalidade de aprendizagem, cada empresa aprende somente aquilo que observa externamente, sem depender de qualquer interação com as outras empresas do arranjo que já inovaram. Com o crescimento desse parâmetro exógeno, aumenta a facilidade e a probabilidade com a qual as empresas do arranjo se aproximam da 'janela tecnológica' e, conseqüentemente, aprendem e inovam. Nesse caso, o percurso temporal da difusão é representado por uma função côncava na qual o número de empresas que inovam aumenta, a uma taxa decrescente, até o ponto de convergir de forma assintótica com o número máximo de empresas potencialmente inovadoras (MAGGIONI; RIGGI, 2002).

Uma terceira hipótese verificada pelos autores afirma que os arranjos 'pequenos', ou ainda nos primeiros estágios de desenvolvimento, podem aprender mais e melhor através das redes sociais amplas e dos canais externos, enquanto que os arranjos 'grandes' ou com bom desenvolvimento (isto é, que apresentem internamente um grau suficiente de variedade de comportamentos necessários à geração da inovação) obtêm resultados melhores através da interação local.

Ainda segundo Maggioni e Riggi, (2002), nas suas primeiras fases de desenvolvimento, um arranjo e sistema produtivo podem aprender, gerar e absorver inovação do externo através dos canais privilegiados, ligados aos empreendedores individuais. Mas, alcançada certa dimensão, ou os mecanismos de transmissão dos conhecimentos se transformam em endógenos, ou, ainda, a capacidade de inovação e de aprendizagem das empresas individuais não conseguem gerir o aumento da dimensão e complexidade do sistema 'maduro'.

Além dessa discussão sobre a difusão em um arranjo ou sistema territorial da inovação, através das modalidades endógenas e exógenas de aprendizagem, os autores atentaram para a seguinte questão: considerando o fato de que uma parte das empresas de um arranjo já tenha adotado uma inovação segundo a modalidade exógena de aprendizagem, como poderia ser facilitado, para as outras empresas, o recurso da modalidade alternativa?

Para os autores, os canais através dos quais podem fluir essas 'externalidades' são diversos: podem ser de tipo institucional e formal, relacionado com o fator comportamental dos agentes como as câmeras de comércio, associações empresariais, autoridades locais. Mas muito mais provável seria o canal do tipo organizacional e 
informal, como aqueles desenhados a partir dos processos de tomada de decisões e de comportamentos das empresas participantes do arranjo e, ainda, através de dinâmicas do tipo imitativas.

Nessas últimas seções discutiram-se as modalidades de aprendizagem e de difusão da inovação no arranjo e sistema produtivo. Nessa ótica, a inovação e a mudança tecnológica e organizacional do inteiro arranjo e sistema produtivo territorial são vistas como fatores propulsores do aumento da produtividade, no caso de inovações organizacionais e de processo, e, do incremento da competitividade, no caso de inovação de produto.

\section{Conclusão}

Este ensaio, ao considerar, como unidade de análise, o contexto dos arranjos e sistemas produtivos territoriais, articulou conceitos, propondo esquemas teóricos que poderão servir de guias para o reconhecimento do complexo fenômeno da aprendizagem e da inovação nestas formas de organização produtiva com foco no território.

Focalizando a base tecnológica dos arranjos e sistemas produtivos foram analisadas as modalidades de aprendizagem e inovação ativadas nestes territórios. Considerando a análise do conhecimento que vem gerado e disseminado nas redes de inovação constituídas entre os atores/agentes, demonstrou-se como essas interações influenciam tanto a criação de conhecimentos como também a sua natureza, gerando, conforme os casos, conhecimentos culturais, tácitos e codificados (ou explícitos).

No âmbito dos arranjos e sistemas produtivos, a criação e a disseminação de conhecimentos ocorrem através de um processo contínuo de integração entre os conhecimentos que fazem parte do patrimônio cultural do território. A proximidade espacial se transforma na chave para a produção, a transferência e o compartilhamento dos conhecimentos tácitos gerados em conjunto, salientando a importância do contexto social e cultural para esse tipo de aprendizado.

Grande parte da literatura insiste em acentuar que, para desenvolver-se um arranjo deve-se balancear dinamicamente o grau de vitalidade interna do seu tecido (sinergia interna) com o de abertura para o mundo (participação em redes globais ou relações externas). Considerando essas discussões, apresentaram-se os resultados de estudos que simularam diversos cenários de interação, através dos dois canais alternativos de aprendizagem coletiva: as conexões internas e as conexões externas, associadas às duas tipologias de conhecimento, com a finalidade de verificar as implicações sobre o processo de criação e difusão da inovação que se nutre do tecido (social, cultural, institucional, tecnológico e produtivo) no qual estão localizadas as empresas.

Essa discussão induz a considerar que, para afrontar o contexto de competição global no qual os mercados e as tecnologias evolvem sempre mais rapidamente os arranjos e sistemas produtivos territoriais, deve-se buscar um equilíbrio entre valorização do conhecimento endógeno e aquisição de conhecimentos exógenos.

A solução que se prospecta parece estar na seleção dos aspectos sociais e da cultura local entendidos como o resultado sinérgico do patrimônio histórico e de conhecimentos tácitos obtidos mediante processos de aprendizagem endógena. E, na seleção dos aspectos da inovação - entendidos como a introdução de novos produtos, processos e métodos organizacionais gerados na interação criativa dos agentes/atores e no recebimento de conhecimentos codificados obtidos através de processos de aprendizagem exógena - aspectos esses considerados úteis para o percurso do desenvolvimento planejado.

Considerando os temas articulados referentes aos processos de aprendizagem, inovação e da dimensão cultural no âmbito dos arranjos e sistemas produtivos territoriais, alguns pontos podem ser enfatizados pela sua importância e pertinência com o campo da administração, entre estes:

- A perspectiva da organização territorial e o papel central da gestão social dos processos de desenvolvimento nas suas diferentes abordagens: econômico, local, social, cultural e humano; 
- A compreensão de questões estratégicas (produtividade e competitividade) e dos processos de aprendizagem e inovação (de produtos, processos e organizacionais), tendo como base a identificação na literatura e a articulação na prática de diferentes focos para abordagem dessas questões: o contexto cultural das organizações territoriais;

Especificamente sobre o descortinar de outras dimensões, como a cultural, no âmbito dos processos de aprendizagem e inovação, construindo sistemáticas que, sem prescindir da complexidade associada, revelam que estas ainda estão evoluindo para incluir aspectos que facilitarão às micro e pequenas empresas aglomeradas a criação e manutenção de vantagem competitiva no contexto dos arranjos e sistemas produtivos territoriais;

- A compreensão de como as redes horizontais de cooperação e competição entre atores públicos e privados, em suas maiorias locais, cria um ambiente favorável ao desenvolvimento de micro e pequenas empresas em condições de competir nos mercados internacionais;

Por fim considera-se, ainda, que esses focos tenham possibilitado a reorientação na formulação de políticas públicas em prol do desenvolvimento local. Garofoli (1999) afirma que o desenvolvimento local está baseado na produção da 'capacidade social', no âmbito da comunidade de empresas e das instituições que operam no território, implicando, ainda, o gradual relacionar-se com os mercados externos e com a produção de conhecimentos e de tecnologia que são produzidas exteriormente ao arranjo ou sistema local. $\mathrm{O}$ desenvolvimento diz respeito à capacidade de inovação e produção da inteligência coletiva, localmente. 


\section{Referências Bibliográficas}

ALBAGLI, S.; BRITO, J. Arranjos Produtivos Locais: Uma nova estratégia de ação para o SEBRAE . Glossário de Arranjos Produtivos Locais. Rede Sist, 2002.

AMIN, A. These are not marshallian times. In: R. CAMAGNI. Innovation networks and spatial perspectives. London: Bellhaven, 1991. p. 105-117.

AYDOLAT, P.; KEEBLE, D. High technology industry and innovative environments: the European experience. GREMI . London: Routledge, 1998.

ARROW, K.J. The economics implications of learning by doing. Review of Economic Studies, 29, 1962. p. 155-173..

BECCATINI, G. Modelli locali di sviluppo. Bologna: II Mulino, 1989.

Mercato e forze locali. II distretto industriale. Bologna: II Mulino, 1987.

BELL, M.; PAVITT, K. The development of technological capabilities. In: Haque, I.U. (ed.) Trade, Technology and international competitiveness. Washington: The World.Bank, 1995. p. 69-1001.

BERETTA, S.; CURINI, L. II ruolo della famiglia nel generare capitale sociale: um approccio di economia política. In: VIII Rapporto CISF sulla famiglia in Itália. Milano: S. Paolo, 2003. p. 290-339..

BRAMANTI, A.; MAGGIONI, M.A. La dinamica dei sistemi produtivi territoriali: teorie, tecniche, politiche. Milano: Franco Angeli, 1997.

BRAMANTI, A.; MIGLIERINA, C. Alle radici della crescita regionale: fattori, fenomeni, agenti. L'Industria, anno XVI, 1, 1995.

BOURDIEU, Pierre. The forms of capital. In: RICHARDSON, J.G. (Ed) Handbook of theory and research for sociology of education. New York: Grenwood, 1985. p.241-58.

BRUSCO, S. The idea of industrial districts: its gênesis. In: F. Pyke, G. Becattini and W. Sengenberger (eds), Industrial Districts and Interfirm Cooperation in Italy. Geneva: International Institute for Labour Staudies, ILO, 1990. p. 10-19.

CALLON, M. The dynamics of techno-economic networs. In: COOMBS, R; SAVIOTII, P.; WASH, V. Technological Changes and Company Strategies: economical and sociological perspectives. London: Harcourt Brace Javanovich Publishers, 1992.

CAMAGNI, R. Innovation networks. Spatial perspectives. London: Belhaven, 1991.

CAMAGNI, R.; CAPELLO, R. . Apprendimento collettivo e competitività territoriale. Milano: Franco Angeli, 2002.

CASSIOLATO, J. E.; SZAPIRO, M. Arranjos e sistemas produtivos e inovativos locais no Brasil. Rede de sistemas produtivos e inovativos locais. Universidade Federal do Rio de Janeiro. Disponivel em <www.ie.ufrj.br/redesist>. Consultado em setembro de 2002.

CHOO, C.W. The knowing organization: how organization use information to construct meaning, create knowledge and take decisions. New York: Oxford University Press, 1998.

COLEMAN, J S. Social Capital in the creation of Human Capital. American Journal of Sociology, v. 94 Suplement, 1988. p. 95-120.

COWAN, R.; DAVID, P; FORAY, D. The explicit economics of knowledge codification and tacitness, 30. TIPIK Workshop, Strasburgo, 1998.

FERRUCCI, L.; VARALDO, R. La natura e la dinamica della impresa distrettuale. L'industria, 80, 1993.

FREEMAN, C. The economics of industrial innovation. Londres: MIT, 1982.

GAROFOLI, G. Lo sviluppo locale: modelli teorici e comparazioni internazionali. Meridiana, 1999 n. 34/35.

IPIRANGA, A.S.R.; AMORIM.A.A.; MOREIRA, M.V. Constructing governance within small firm cluster: a view from the developing world. In: Buildind a sustainable city through sustainable enterprise. Chicago - USA: BELL CONFERENCE IIT - Stuart Graduate School of Illinois, 2004.

JOHNSON, B.; LUNDVALL, B. Why all this fuss about codifiedknowledge?, DRIUD Winter Conference, january, 2001. p.18-20. KIM, D. The link between individual and organizational learning. Sloan Management Review. v. 35, p. 37-50, Fall. 1993 
LASTRES, H. M.M.; VARGAS, M. A.; LEMOS, C. Novas políticas na economia do conhecimento e aprendizado. Disponivel em <www.ie.ufrj.br/redesist>. Consultado em 29 de novembro de 2003.

LIPPARINI, A.; LORENZONI, G. Le organizzazione ad alta intensità relazionale. Riflessioni sui processi di learning by interacting nelle aree ad alta concentrazione di imprese. L'industria, a. XVII, 4, 1996.p. 817-839.

LIPPARINI, A.; LOMI, A. Interorganizational relations in the Modena biomedical industry. A case study in local economic development. In: A. GRANDORI, A. Interfirm network organisation and industrial competition, London: Routledge, 1998.

LORENZONI, G. L'architettura di sviluppo delle imprese minori. Bologna: II Mulino, 1990.

LORENZONI, G. Accordi, reti e vantaggio competitivo. Milano: Etas, 1992.

LUNDVALL, B.A. National Systems of Innovation; towards a theory of innovation and interactive learning. London: Pinter Publishers, 1992. Innovation growth and social cohesion: the danish model. Chetenham, UK: Edward Elgar, 2002.

LUNDVALL, B.A.; MASKELL, P. Nation states and economic development - from national systems of production to national systems of knowledge creation and learning. In: MAILLAT, D.; QUEVIT, L.; SENN, L. Reseaux d'innovation et mileux innovateurs: un pari le dévelopment régional, 1, Neuchatel, 1993.

MAGGIONI, M.A.; BRAMANTI, A. Local and global networks in the economics of SMEs. Is proximity the only thing that matters? In: NAUGHTON, R. Mc; GREEN, M. Global competition and local networks. London: Ashgate, 2000.

MAGGIONI, M.A.; NOSVELLI, M. La cultura dei sistemi produttivi territoriali tra formazione e innovazione. In: Osservatorio Impresa e Cultura, Cultura e competitività. Per um nuovo agire imprenditoriale. Roma: Rubbettino, 2003.

MAGGIONI, M.A.; RICCI,M. Forme alternative di collective learning: um approccio sistemico-popolazionista ed alcune simulazioni:" In: CAMAGNI, R.; CAPELLO, R. Apprendimento colletivo e competitività territoriale. Milano: Franco Angeli, 2002.

MASKELL, P.; MALMBERG, A. Apprendimento localizzato e competitività. In: BRAMANTI, A.; MAGGIONI, M. ${ }^{a}$ La dinamica dei sistemi produttivi territoriali: teorie, tecniche, politiche. Milano: Franco Angeli, 1997.

MARSHALL, Alfred. Princípios de Economia. São Paulo: Nova Cultural, 1996.

MESSNER, D.; MEYER-STAMER, J. Governance and Networks. Tools to study the dynamics of clusters and global value chains. Paper prepared for the IDS/INEF Project The Impact of Global and Local Governance on Industrial Upgrading. University of Duisburg, 2000.

NONAKA, I.; TAKEUCHI, H. The knowledge-creating company: how Japanese companies create the dynamics of innovation. New York: Oxford University Press, 1995.

NONAKA I.; TOYAMA, R.; KONNO, N. SECI, ba and leadership: a unified model of dynamic knowledge creation. In: LITTLE, S.; QUINTAS, P.; RAY, T. (Eds.). Managing Knowledge an Essential Reader. London: Sage Publications, 2002.

PIORE, M.G.; SABLE,C.F. The second industrial divide. New York: Basic Books, 1984.

POLANYI, M. The tacit dimension. Gloucester: Peter Smith, 1966.

Personal Knowledge: toward a post-critical philosophy. Chicago: The University Chicago Press, 1958.

PUTNAM, R.D. Comunidade e democracia: a experiência da Itália moderna. Rio de Janeiro: Editora Getúlio Vargas, 1996.

POWELL, W.W.; KOPUT, K.W.; SMITH-DOERR, L. Inter-organizational collaboration and the locus of innovation: networks of learning in biotechnology. Administrative Science Quarterly, v.41, n. 1, 1996. p. 116-145.

RATI, R.; BRAMANTI, R.; GORDON, R. The dynamic of innovative regions: the GREMI approach. Aldershot: Avebury, 1997.

ROSENBERG, N. Learning by using. In: Inside the black box: technology and economics. Cambridge: Cambridge University Press, 1982. p. 102-140.

ROTHWELL, R. Industrial, innovation: success, strategy, trends. In: DODGSON, M.; ROTHWELL, R. The handbook of industrial innovation. Cheltenham: Edward Elgar, 1995. 
STIGLITZ, J.E. Learning to learn, localized learning and technological progress. In: DASGUPTA, P.; STONEMAN, P. Economic, policy and technological performance. Cambridge: Cambridge University Press, 1987.

THROSBY, D. Economics and Culture. Cambridge: Cambridge University Press, 2001.

VASCONCELOS, F.C. Da gestão do conhecimento à gestão da ignorância: uma visão co-evolucionária. In: Revista de Administração de Empresas - RAE, v.1, n.4, São Paulo, out./dez. 2001.

VEDOVELLO, C.; MELO, M.S. de; MARIUS, L.M. Globalização de Competências Inovadoras e o Papel de Infra-Estruturas Tecnológicas: evidências de Institutos de Pesquisa e Desenvolvimento (PCtD) em Telecomunicações no Brasil. In: XXVIII ENCONTRO DA ASSOCIAÇÃO NACIONAL DOS PROGRAMAS DE PÓS-GRADUAÇÃO - ENANPAD, 2004, Curitiba. Anais... Curitiba: ANPAD, 2004.

ZARIFIAN, P. Objetivo competência: por uma nova lógica. São Paulo: Atlas, 2001.

1 Arranjos produtivos locais podem ser definidos como sendo "aglomerações territoriais de agentes econômicos, políticos e sociais, com foco em um conjunto específico de atividades econômicas e que apresentam vínculos e interdependência" (ALBAGLI; BRITO, 2002:3).

2 Sistemas produtivos e inovadores locais são definidos como "arranjos produtivos cuja interdependência, articulação e vínculos consistentes resultam em interação, cooperação e aprendizagem, possibilitando inovações de produtos, processos e formatos organizacionais e gerando mais competitividade empresarial e capacitação social" (ALBAGLI; BRITO, 2002:3). 